\title{
Analysis of Information Security Protection Strategy for Network Electronic Engineering Archives
}

\author{
Chu Xue \\ Qilu Normalu University, Jinan, Shandong, 250200
}

Keywords: Electronic Engineering Archives; information security; computer technology

\begin{abstract}
With the development of the country and the progress of the society, our country has begun to apply the corresponding electronic information technology in many industries and management. In this process, the technology also highlights its obvious advantages. Compared with the paper documents, the application of electronic information technology in the management and transmission of documents can save more resources and space, and to a large extent, reduce the use of human resources. At present, in our institutional units, the network electronic engineering archives technology has been widely used. The advantages of this technology are self-evident, but it has a more obvious dual nature, and there are many problems and loopholes in security, which seriously endangering the interests of the citizens and the state. Therefore, in the future development, it is necessary to strictly control the safety and standardization of its development so that it can further achieve the development goals. This paper has carried on the detailed discussion to this question, and has proposed the reasonable optimization measures to the corresponding question.
\end{abstract}

In the process of China's development, electronic information technology has played an important role in promoting the development of electronic information, and the security of electronic information has also been put forward in the process of development. Therefore, in the follow-up development, it is essential to strengthen the control system of electronic information security, and deepen the work of the system. In this paper, the related concepts of electronic engineering archives and its influencing factors are the breakthrough points, and the detailed description is made according to its specific content, which provides effective optimization measures for the development of the electronic engineering archives information security in China.

\section{Related Concepts and Influencing Factors of Electronic Engineering Archives}

The development of computer technology is mainly from 90 years since twentieth Century. After this period, the electronic engineering archives technology has gradually entered the people's vision, and in the follow-up development, it is closely related to the human life. The network electronic engineering archives mainly refers to the combination of various kinds of electronic images and information in the Internet technology. In the process of its operation, the is stored or analyzed with advanced computer equipment, electronic information technology and so on. And be able to identify, correspond, and link with the documents and documents related to individuals or enterprises. As far as the current development technology is concerned, the complete archival information of electronic engineering should have a variety of existing forms such as data, database and multimedia data. According to the needs, the storage and electronic equipment should not be processed manually. In addition, the total storage of electronic engineering files is large, far beyond paper. Quality file information. However, in its operation process, mainly rely on computer technology, Internet, electronic information technology, and so on, once the corresponding technology, platform failure, electronic engineering file information can not play its effect.

In modern science and technology society, electronic documents mainly refer to the form of digital or data in the high and new data equipment or the corresponding environment, and store the data and graphic data of the corresponding storage carriers such as disc, hard disk, disk and so on. In daily life, this kind of files can be read, analyzed and processed by computer technology, and can 
be transmitted on mobile devices such as mobile phones. Compared with paper files, the form has a larger storage space, and it is easier to save, handle and transmit. The specific characteristics are as follows: (1) strong dependence on the corresponding software and hardware. Electronic Engineering Archive Information and other electronic documents are done by digital equipment such as computers. In the course of operation, this kind of equipment will depend on the corresponding software and hardware conditions. In the early stage of the rise of computer technology, the technology in this industry does not have comprehensive skills. Therefore, in the process of updating the technology, it will cause damage to the existing data, and make it partially missing or completely damaged; (2) the corresponding electronic files can not be read directly. Staff and other related personnel can not read them directly when they are reading the data, and other equipment or software facilities are needed; (3) the related data information is separated from the information carrier. In the traditional paper documents, the document information is unify with the paper. However, electronic files, such as electronic engineering files, are in a large degree of separation from the equipment carrier in the actual work, which is a hidden danger for the security problems. (4) extremely convenient sharing features and more difficult security maintenance. With the simultaneous existence of technology and carrier, electronic documents such as electronic engineering archives and information can be shared conveniently and quickly. However, in this environment, the security problem is more obvious. Relatively speaking, the maintenance of related work is more complicated.

\section{Existing Problems in Information Security Maintenance of Network Electronic Engineering Archives}

There are two sides of all things in the world, and the Internet technology is no exception. At the same time, it has more obvious related security problems while realizing convenient and rapid development. The main reason is that the data information in the transmission, is the corresponding segmentation, in the process, each segment is likely to be intercepted, read or malicious destruction by the unlawfully, the hacker in the attack, the main application of the computer system in the creation, for the convenience of system maintenance back door entry The most serious attack can change the most core and most valuable functional part in the computer operating system. However, from the current development of China's computer, due to the late start, the safety protection work is not enough, so that it has a more obvious lag. Therefore, in terms of network system security maintenance, we need to further improve relevant strategies and technologies.

Electronic files, such as electronic engineering files, are mainly stored in the network cloud or like computer hard disk. It is convenient and extremely flexible when changing or changing it. In the process, no obvious change marks are left, and it is not easy to be detected. Although the characteristics provided convenience for the work of the staff, they also created favorable conditions for the lawless elements. Therefore, electronic records are largely untrusted or trusted by a large extent. In addition, in the transmission or preservation of electronic engineering files, it is very easy to be infected or destroyed by various virus programs in the network, which may cause the missing or breakage of the information files, resulting in the loss of the original value and the lack of the corresponding credibility in the application. At the same time, in the work, in the work, the electronic engineering archives are separated from the carrier carrier in the operation process. At this time, the corresponding electronic files can be transferred. In the process of transmission, the true and false characteristics of a variety of information in the network are difficult to distinguish.

In terms of the current development of China's electronic information technology, there are two major problems in the organization and management. The specific manifestations are: first, the relevant laws and regulations on electronic information technology are not sound. In the past few years, the relevant departments of the state and their leaders have put forward a corresponding department. The rules and regulations are listed, but the management of this technology application is relatively unfamiliar in China. Therefore, when the corresponding system is established, there will be a big mistake. For example, the rules related system is too general, the management rules are too broad and do not conform to the actual application system. In addition, when the corresponding 
work is carried out, the effect is not strong and there is no more obvious pertinence, which is the related safety management worker. The work of the staff adds to the difficulty. Two, the comprehensive quality of the related staff is poor. In the development of today's technology, most of the relevant units in the current development of our country pay more attention to the construction of the corresponding software and hardware in the development and operation of the enterprises, and to a certain extent ignore the safety management of the related applications. Therefore, the staff in this kind of enterprise have excellent skills in the development and application of hardware and software. However, there are no corresponding skills and skills in the security protection of the system. Moreover, the staff of electronic engineering file management do not have comprehensive knowledge and related ability for computer related problems. Therefore, during its work, it is impossible to accurately and thoroughly manage the electronic engineering related archives management, and make it more serious. At the same time, the administrators of electronic archives lack the awareness of the safety management of the relevant archives information in their daily work, but only exercise the management rights of guarding and keeping them, and can not have network protection function.

\section{Strategies to Optimize the Information Security Protection of Network Electronic Engineering Archives}

In the overall optimization of the work of network electronic engineering archives information security protection, first of all, it is essential to set up a sound concept of safe archives to ensure the safety of electronic engineering archives. In the new social environment and development state, the relevant units of the archival work should ensure the interests of our citizens and the state in the process of carrying out the reform work, and on this basis recognize the importance of the work content, and actively correspond to the call and development side of the relevant policies and departments of the country. The needle. In carrying out the relevant work, we should focus on training the awareness of network security, and combine the relevant policies and laws of the electronic engineering archives information security. In the actual work, we should strengthen the maintenance and management of the network security, and carry out corresponding and foreign technology based on its specific development. Actively learn from and study, strengthen the security situation of the network environment, make the network electronic engineering file information security can have a more perfect working environment, and further guarantee the security of the country and the citizens [4].

Develop backup habits in work and strengthen related safety protection work. The staff of the electronic engineering archives information security management have great risks in the transmission and storage of the files, so they should cultivate more rigorous work habits. For example, in the work, we should strengthen the backup habit of self generation, keep the files in shape, and find the relevant problems effectively and make a timely and reasonable solution when dealing with the corresponding problems. In addition, in the process of backup, we should record the backup staff and the specific backup time in detail, and check and collate it with periodic work as the criterion, in order to ensure the safety of the electronic engineering archives information in addition, when carrying out related work, staff should strengthen the awareness of safety protection of electronic engineering archives information, and make specific and effective implementation measures. Specific practice, first, in carrying out the work, we should strictly follow the state regulations, use the high quality electronic files for the storage of data, and carefully review the stored materials to ensure the compatibility of the two, and can enable the two to better use the other side, and on this basis can extend the life of electronic files. The two is to create a system and a safe physical environment for the electronic engineering files and other related facilities and technology, so that it will not be damaged by temperature, humidity and other physical damage in the operation process, and finally achieve better work effect. Three, when the relevant departments of the electronic engineering archives information management are working, we should build a healthy body. The whole internal management mechanism makes it possible to limit and manage the contents of the archives to a great extent, so that the staff can make clear division of labor, and 
strictly complete their respective responsibilities under the clear division of labor, and finally achieve the goal of improving the security protection of the network electronic engineering archives information [6].

Establish a sound regulatory mechanism for archival information system. For the present era, it is one of the key goals of the unit meeting organization to establish an authoritative archival management working environment and make it able to carry out a more systematic security management function to a large extent. In the actual working environment, the relevant departments and departments should strengthen the relevant system construction and the quality requirements for the staff. First of all, due to the lack of specific and specific relevant laws and regulations on the work content of electronic engineering file information security protection, it can not be effectively solved by technology. Therefore, the first problem to be solved is to establish a sound supervision mechanism for archival information system, so that it can help solve such problems to a large extent. Secondly, the related staff lack the knowledge of computer technology. In the process of their work, the knowledge of professional skills related to work is less, and the corresponding safety management can not be carried out in their work, which seriously affects the development of safety management. Therefore, the unit or organization should strengthen the skill requirements of the staff and train them regularly so that they can improve their self work to a large extent. Finally, in the work, the staff has no comprehensive understanding of the importance of the safety of electronic engineering files, which will seriously impede the enthusiasm and seriousness of their work. Therefore, in the actual work, the leadership should strengthen the management of education so that they have more accurate positioning for their own work and actively invest in their own work. In the work of the body [5].

\section{Conclusion}

In summary, the main problems existing in the development of Electronic Engineering Archives in China are the hidden security problems in the Internet, the convenience and flexibility of electronic engineering files in the change of electronic engineering and the poor organization and management of the network electronic engineering files, and the specific problems are raised in view of the specific problems. There are three measures to improve the Internet security protection system, to train the backup habits in the work, to strengthen the relevant safety protection work and to establish a sound supervision mechanism of the archives information system. The research of this paper can further promote the development of the construction of the electronic engineering archives information security in our country.

\section{References}

[1] Li H J, Wang X. Research on Information Security Analysis and Protection Strategy of Computer Network[J]. Information Security \& Technology, 2016.

[2] Yuan X. Analysis of computer network information security and protection strategy[J]. Wireless Internet Technology, 2016.

[3] Wang H, Deng W. Analysis of effective protection strategy for computer network information security[J]. Jiangsu Science \& Technology Information, 2016.

[4] Shi J, Li J. The Security and Protection Strategy Study of Computer Network Information[C]// International Conference on Electronics, Network and Computer Engineering. 2016.

[5] Zhang C. Study on Security and Protection Strategy of Computer Network Information[C]// International Conference on Electronics, Network and Computer Engineering. 2016. 